\title{
MLKL is a potential prognostic marker in gastric cancer
}

\author{
WEI SUN ${ }^{1}$, WENYAN YU ${ }^{2}$, LILI SHEN ${ }^{3}$ and TIEAO HUANG ${ }^{1}$ \\ ${ }^{1}$ Department of General Surgery, Xiangcheng People's Hospital, Suzhou, Jiangsu 215131; \\ ${ }^{2}$ Department of Oncology, The First Affiliated Hospital of Soochow University, Suzhou, Jiangsu 215006; \\ ${ }^{3}$ Department of Oncology, Haimen People's Hospital, Haimen, Jiangsu 226100, P.R. China
}

Received November 25, 2018; Accepted June 13, 2019

DOI: $10.3892 / \mathrm{ol} .2019 .10687$

\begin{abstract}
The mixed lineage kinase domain-like protein (MLKL), which is a major mediator of the necroptosis pathway, is involved in a certain cancers. The present study aimed to explore the expression patterns and exact role of MLKL in gastric cancer (GC) tumorigenesis and progression. In Cancer Cell Line Encyclopedia analysis, the MLKL mRNA expression levels in GC cell lines were not higher compared with that in other cancer cell lines. The results of the present study demonstrated that MLKL expression was decreased in gastric cancer tissues compared with that in normal tissues. In the Kaplan-Meier Plotter database survival analyses, decreased MLKL expression was associated with poor overall survival and first progression in patients with gastric cancer. In Oncomine gene co-expression analysis, MLKL expression was significantly associated with fatty acid 2-hydroxylase (FA2H) expression, which also exhibited similar effects on the prognosis of patients with GC in the survival analysis. This result suggested that FA2H may be a downstream molecule of MLKL. The results of the present study indicated that MLKL may be a novel prognostic biomarker for patients with GC.
\end{abstract}

\section{Introduction}

Cancer is the leading cause of mortality worldwide; $1,762,450$ new cancer cases and 606,880 cancer mortalities were estimated in 2019 (1). Furthermore, 27,510 new cases of gastric cancer leading to 11,140 deaths are expected in the United States in 2019 (1). The incidence of GC is particularly high

Correspondence to: Dr Lili Shen, Department of Oncology, Haimen People's Hospital, 253 People's West Road, Haimen, Jiangsu 226100, P.R. China

E-mail: shenlilimao2012@163.com

Dr Tieao Huang, Department of General Surgery, Xiangcheng People's Hospital, 1060 Hua Yuan Road, Suzhou, Jiangsu 215131, P.R. China

E-mail: huangtieao@126.com

Key words: gastric cancer, mixed lineage kinase domain-like protein, fatty acid 2-hydroxylase, prognosis, necroptosis in eastern Asian countries, such as China, Japan and South Korea (2). In China, GC is the second leading cause of cancer-related morbidity and mortality (3). Despite the developments in multimodal therapy strategies, such as surgery, chemotherapy, radiotherapy, target therapy and immunotherapy, the prognosis of advanced GC remains poor (4). Thus, identifying novel molecular biomarkers for GC is crucial to the improvement of the therapeutic effects and of the survival of patients with GC.

The mixed lineage kinase domain-like protein (MLKL) is a component of the receptor-interacting kinase 1 (RIP1)/ receptor-interacting kinase 3 (RIP3)/MLKL pathway, which is involved in cell necroptosis $(5,6)$. Necroptosis, which is a form of programmed necrotic death, is characterized by increased cell volume, cell rounding, nuclear membrane dilation, chromatin condensation, cytoplasmic membrane disruption, organelle swelling, and inadequate caspase activation (6-11). The interaction between RIP3 and RIP1 recruits the downstream effector MLKL protein to form necrosomes, where MLKL is phosphorylated $(5,12)$. MLKL depletion in cancer cells induces spontaneous phosphorylation of H2A histone family member X, an early marker for DNA damage, which suggests that MLKL may serve a crucial role in response to DNA damage (13). Previous studies have demonstrated that MLKL serves as a potential prognostic biomarker for patients with multiple carcinomas (14-17). These data suggest that MLKL may serve a distinctive role in certain cancers, including GC. However, systematic studies on MLKL expression and its prognostic value in human cancers are still insufficient.

In the present study, the expression of MLKL in GC samples and normal tissues was evaluated, the association between clinicopathologic and prognostic parameters in patients with GC was assessed in a large database, and a potential underlying mechanism was investigated.

\section{Materials and methods}

Oncomine database analysis. Oncomine database (http:// www.oncomine.org), a publicly accessible online cancer microarray database, was used to analyze MLKL mRNA expression levels in tumor and normal tissues. The thresholds were set as follows: the fold change was defined as 2 and $\mathrm{P}$-value was set to 0.01 . Cancer types, genes, datasets, sample sizes, fold changes, t-test results and P-values were obtained from studies that showed statistically significant differences. 
Cancer Cell Line Encyclopedia (CCLE) analysis. The CCLE database (https://portals.broadinstitute.org/ccle) is an online compilation of gene expression, chromosomal copy number and parallel sequencing data from 947 human cancer cell lines. The CCLE database was used to analyze the mRNA expression levels of MLKL in a series of cancers. Information about cancer types, genes, datasets, sample sizes, fold changes, t-test results and P-values were collected.

Kaplan-Meier (KM) Plotter database analysis. The KM Plotter (http://kmplot.com/analysis) was used to evaluate the prognostic values of MLKL and fatty acid 2-hydroxylase (FA2H) in GC. The data from 882 patients with GC were obtained from the KM database (tumor-node-metastasis (TNM) stages: T1, $\mathrm{n}=14 ; \mathrm{T} 2, \mathrm{n}=253 ; \mathrm{T} 3, \mathrm{n}=208 ; \mathrm{T} 4, \mathrm{n}=39$; N0, $\mathrm{n}=76 ; \mathrm{N} 1+2+3, \mathrm{n}=437 ; \mathrm{M} 0, \mathrm{n}=459 ; \mathrm{M} 1, \mathrm{n}=58$; surgical treatment only, $\mathrm{n}=393$ ). Using the selected parameters, the analysis was performed on the data from 631 patients with GC for overall survival (OS) analysis and from 522 patients in first progression (FP) analysis. The patients were split into high and low expression groups according to the median values of mRNA expression, and Kaplan-Meier survival plots were obtained. Logrank test $\mathrm{P}<0.05$ was considered to indicate a statistically significant difference. Survival outcome, hazard ratios (HR), 95\% CI and P-values were summarized from the KM plotter webpage.

Patient samples. A total of 25 patients with histopathologically confirmed GC and complete follow-up data were recruited between March and September 2017 from the Xiangcheng People's Hospital. The inclusion criteria were as follows: i) Histopathological diagnosis of adenocarcinoma; ii) patients who had not received anti-tumor treatment prior to the study; and iii) computed tomography of the chest, abdomen and pelvis did not show evidence of distant metastasis. The exclusion criteria were as follows: i) Patients who cannot tolerate surgical treatment; and ii) patients who did not allow specimen provision. There were 17 men and 8 women, aged between 38 and 71 years (median age, 53 years). Written informed consent was obtained from all patients and the protocol of the study was approved by the Institutional Review Board of Suzhou University (Suzhou, China; approval no. 2016958021; 5 March 2016). Fresh gastric cancer and normal tissues $(\sim 2 \mathrm{~cm}$ from the tumor) were collected from the patients, immediately stored in liquid nitrogen, and lysed with TRIzol ${ }^{\circledast}$ (Invitrogen; Thermo Fisher Scientific, Inc.). None of the patients had received chemotherapy or radiotherapy prior to surgery. Pathological variables including depth of invasion, lymph node metastasis, distant metastasis, TNM stage and anti-tumor therapy were obtained. The Cancer Staging Manual of the American Joint Committee on Cancer (version 8) (18) was used for patient staging.

Reverse transcription-quantitative PCR (RT-qPCR). RT-qPCR was performed as described previously (19). Total RNA was isolated from frozen GC and normal tissues with TRIzol ${ }^{\circledR}$. Complementary DNA was prepared using oligo(dT) primers with Primer Script RT Mix (Takara Biotechnology Co., Ltd.) according to the manufacturer's protocol. Expression levels of MLKL mRNA were determined with SYBR-Green PCR kit
(Takara Biotechnology Co., Ltd.) in an ABI PRISM 7500 fast Sequence Detection System (Applied Biosystems; Thermo Fisher Scientific, Inc.). RT-qPCR reactions were performed as follows: $95^{\circ} \mathrm{C}$ for $20 \mathrm{sec}, 95^{\circ} \mathrm{C}$ for $10 \mathrm{sec}$ and $60^{\circ} \mathrm{C}$ for $45 \mathrm{sec}$ for 40 cycles. The relative expressions levels were expressed as $2^{-\Delta \Delta \mathrm{Cq}}(20)$ using $\beta$-actin as the reference gene. Real-time PCR primers were as follows: MLKL forward, 5'-TTCACCCAT AAGCCAAGGAG-3' and reverse, 5'-GGATCTCCTGCATGC ATTTT-3'; and $\beta$-actin forward, 5'-CTCCATCCTGGCCTC GCTGT-3' and reverse, 5'-GCTGTCACCTTCACCGTTCC-3'.

Statistical analysis. Statistical analyses were performed with GraphPad Prism 6 (GraphPad Software, Inc.) and SPSS Statistics 19.0 (IBM Corp.). Student's paired t-test was used to compare the expression levels of MLKL between gastric cancer and normal tissues. $\mathrm{P}<0.05$ was considered to indicate a statistically significant difference. Data are presented as the mean \pm standard deviation.

\section{Results}

MLKL mRNA expression levels in human cancers. To assess the MLKL mRNA expression levels in tumor and normal tissues in multiple cancers, the Oncomine database was used. The database contained 257 unique analyses and 504 unique analyses without outliers (Fig. 1A). In 77 of the 504 unique analyses without outliers, 67 revealed that MLKL mRNA expression levels were lower in tumors compared with normal tissues, whereas 20 analyses indicated an opposite result. Only one study revealed an increased MLKL mRNA expression level in GC. In addition, MLKL mRNA expression levels in GC and normal tissues were determined in a cohort of 25 patients by RT-qPCR; the results indicated that MLKL mRNA levels were significantly lower in tumors compared with those in normal tissues $(\mathrm{P}<0.001$; Fig. 1B). Additionally, CCLE analysis demonstrated that MLKL was not upregulated in GC cell lines (Fig. 2).

Prognostic effects of MLKL mRNA expression in patients with $G C$. Patients with GC with high MLKL mRNA expression levels exhibited improved $\mathrm{OS}$ (HR=0.80; $\mathrm{P}=0.045$; Fig. 3A) and FP (HR=0.70, p=0.0036; Fig. 3B). In addition, the prognosis of patients with high and low MLKL expression levels in different $\mathrm{T}$ (depth of invasion), $\mathrm{N}$ (lymph node metastasis) and $\mathrm{M}$ (distant metastasis) stages and in patients receiving surgical treatment was evaluated. In T2 (OS, HR $=0.56, \mathrm{P}=0.0066$; FP, HR=0.56, $\mathrm{P}=0.0066$ ), N0 (OS, HR=0.36, $\mathrm{P}=0.011$; FP, $\mathrm{HR}=0.37, \mathrm{P}=0.013), \mathrm{N} 1+2+3(\mathrm{OS}, \mathrm{HR}=0.7, \mathrm{P}=0.012 ; \mathrm{FP}$, $\mathrm{HR}=0.7, \mathrm{P}=0.0055), \mathrm{M} 0$ (OS, HR=0.7, $\mathrm{P}=0.011 ; \mathrm{FP}, \mathrm{HR}=0.69$, $\mathrm{P}=0.0055)$, M1 (OS, HR=0.42, $\mathrm{P}=0.016 ; \mathrm{FP}, \mathrm{HR}=0.43$, $\mathrm{P}=0.026)$ and patients receiving surgical treatment alone (OS, HR=0.69, $\mathrm{P}=0.01$; FP, HR=0.64, $\mathrm{P}=0.0013$ ), high MLKL expression was significantly associated with longer OS and longer FP compared with patients with low MLKL expression. However, in T3, no significant association between MLKL and the prognosis of patients with $\mathrm{GC}$ was observed $(\mathrm{OS}, \mathrm{HR}=0.77$, $\mathrm{P}=0.14 ; \mathrm{FP}, \mathrm{HR}=0.79, \mathrm{P}=0.16$ ).

Co-expression analysis of MLKL and FA2H in tumors. The co-expression of MLKL and other genes were investigated by 
A

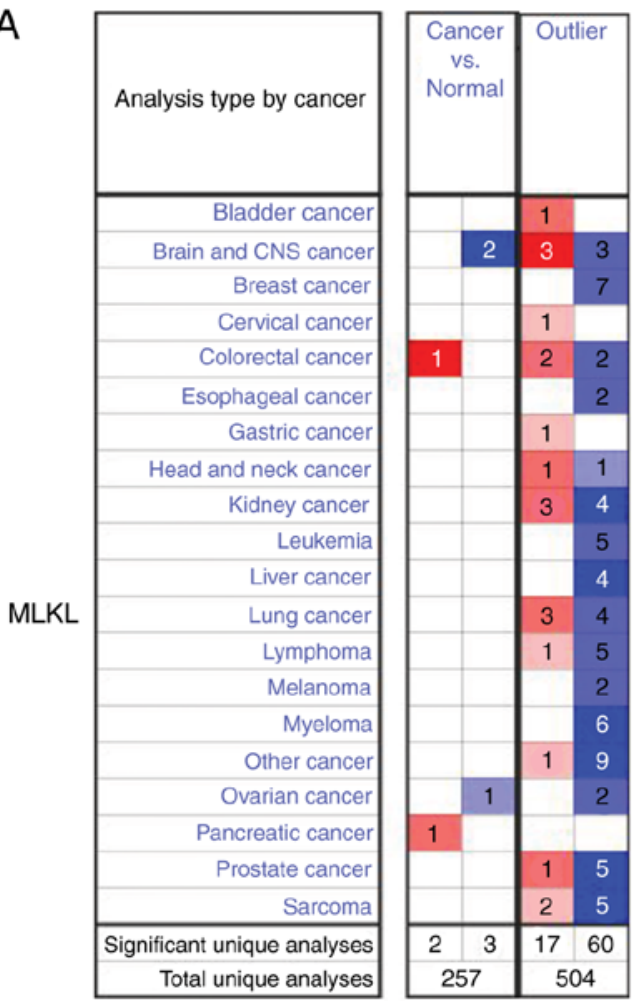

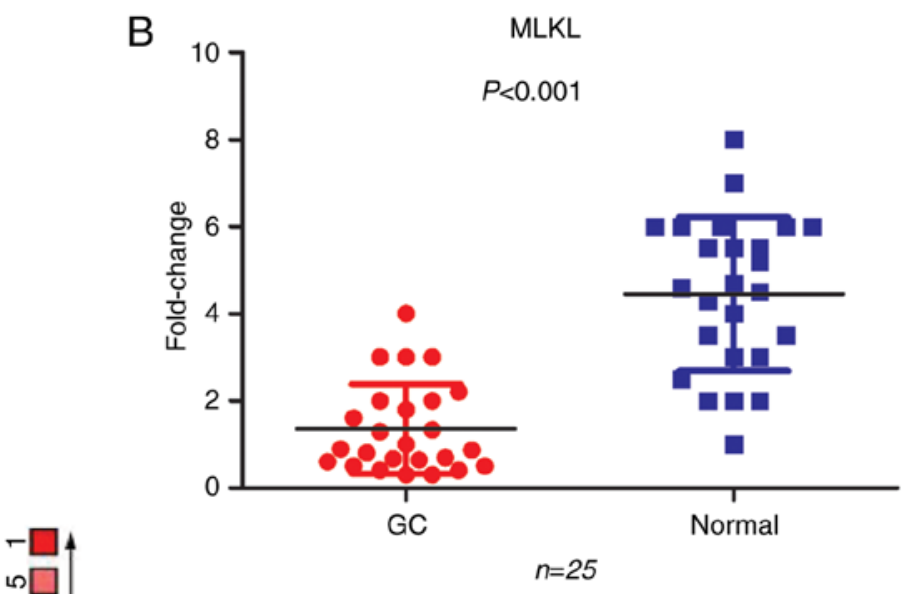

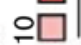

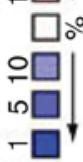

Figure 1. mRNA expression pattern of MLKL in different tumor types. (A) A table demonstrating numbers of datasets with statistically significant MLKL mRNA upregulation (red) or downregulation (blue) in cancer compared with normal tissue. The P-value threshold was set to 0.01 . The number in each cell represents the number of analyses that met the threshold within the analysis and cancer types. (B) MLKL expression levels in tissue samples obtained from patients with GC. MLKL mRNA levels were significantly lower in GC compared with normal tissues $(\mathrm{P}<0.001)$. MLKL, mixed lineage kinase domain-like protein; GC, gastric cancer.

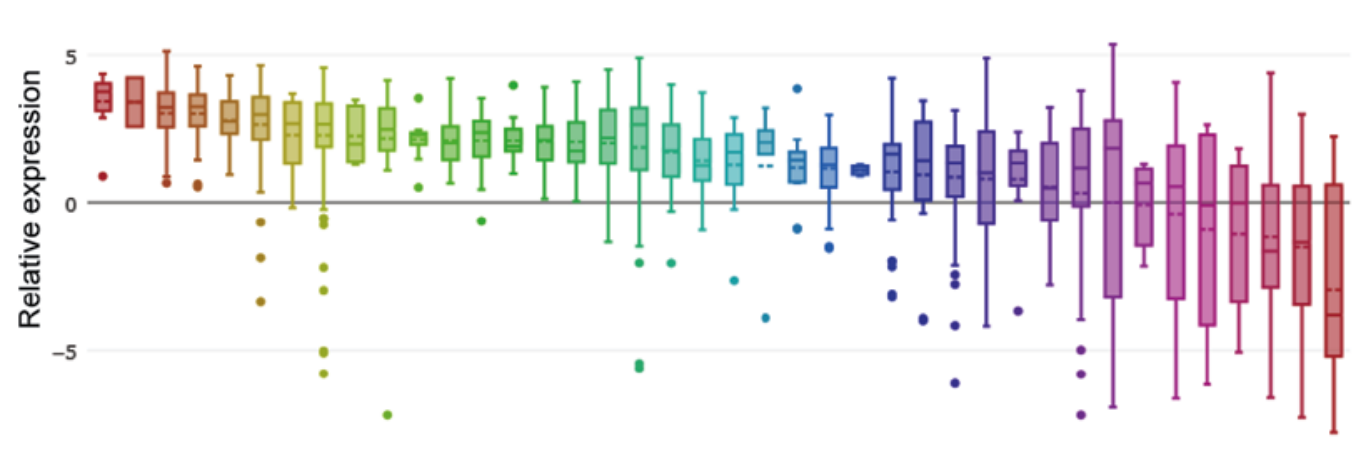

$-10$

$\square$ T-cell_lymphoma_other(11)
meningioma(3)
$\square$ pancreas(46)
$\square$ AML(39)
$\square$ kidney(37)
$\square$ colorectal(63)
bile_duct(8)
$\square$ lung_NSC(136)
$\square$ leukemia_other(5)
$\square$ esophagus(27)
$\square$ thyroid(12)
$\square$ lymphoma_Hodgkin(13)
$\square$ urinary_tract(28)
$\square$ T-cell_ALL(16)
$\square$ upper_aerodigestive(33)
$\square$ CML(15)
$\square$ multiple_myeloma(29)
stomach(39)
glioma(66)
$\square$ Ewings_sarcoma(12)
B-cell_al(13)

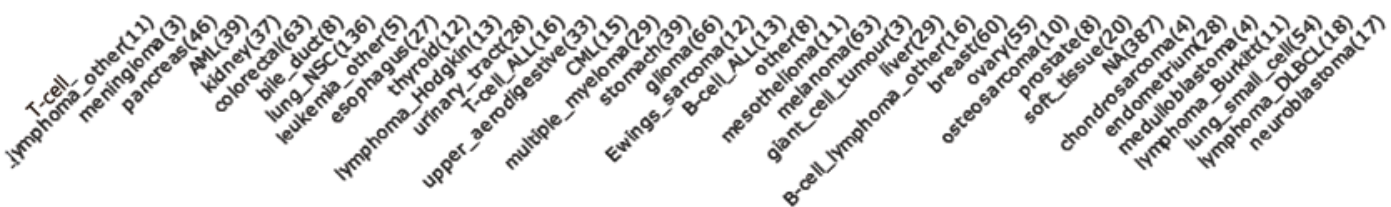

Figure 2. MLKL expression in a range of cancer cell lines from the Cancer Cell Line Encyclopedia analysis. The mRNA expression level of MLKL was not high in gastric cancer compared with other cancer cell types. MLKL, mixed lineage kinase domain-like protein.

using Jimeno Pancreas, Ooi gastric and Collisson CellLine from the Oncomine database. MLKL expression significantly correlated with FA2H in a number of tumors (Fig. 4). In the survival analysis, high FA2H expression was associated with 
A

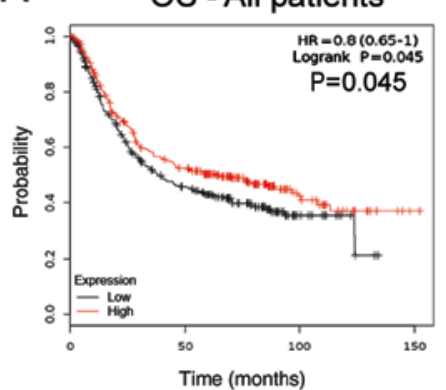

OS - All patients

$\mathrm{OS}-\mathrm{N} 1+2+3$

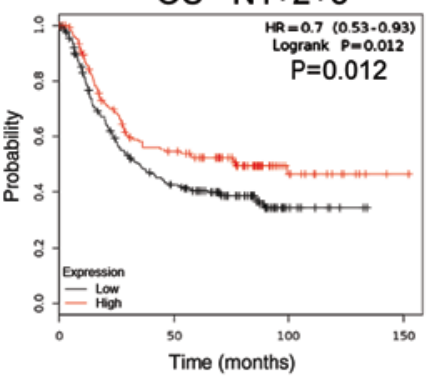

B

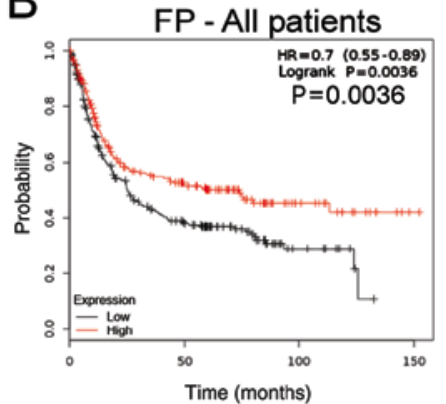

$\mathrm{FP}-\mathrm{N} 1+2+3$

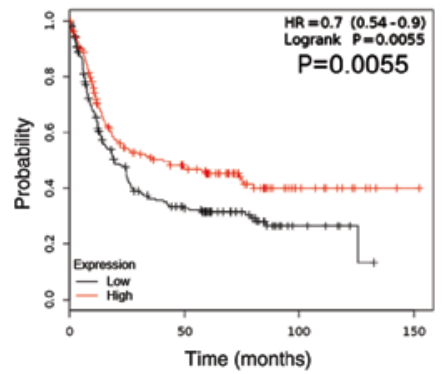

OS - MO

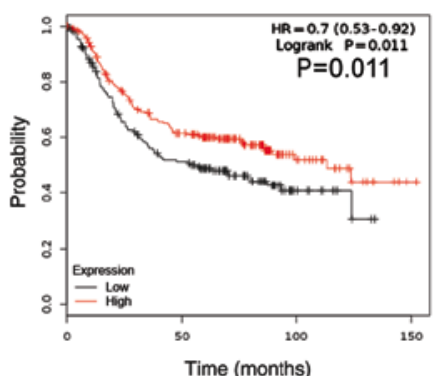

OS - T2

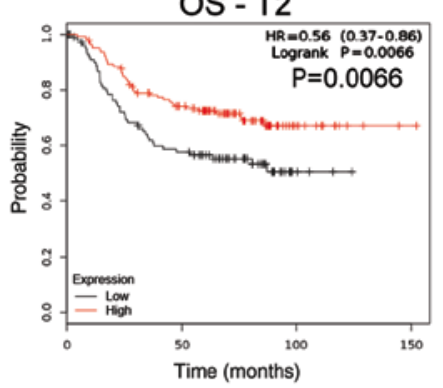

FP - MO

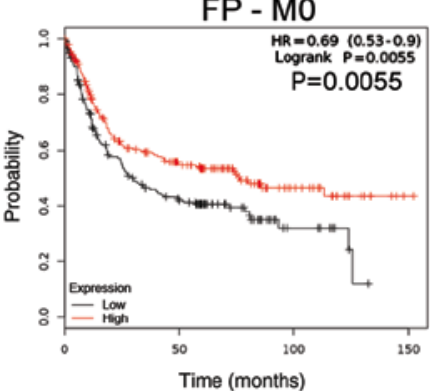

FP - T2

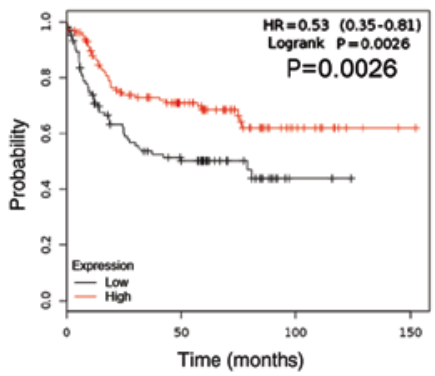

OS - M1

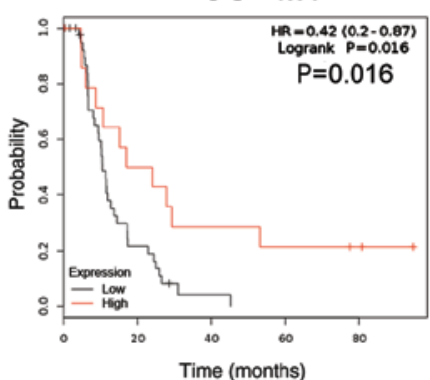

OS - T3
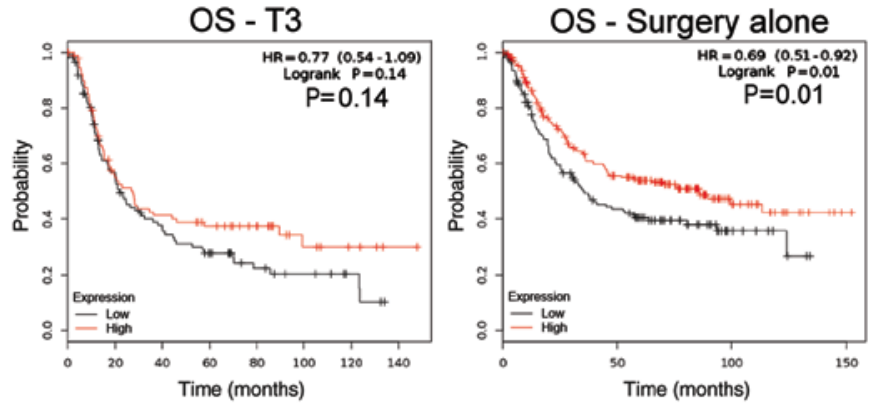

OS - NO

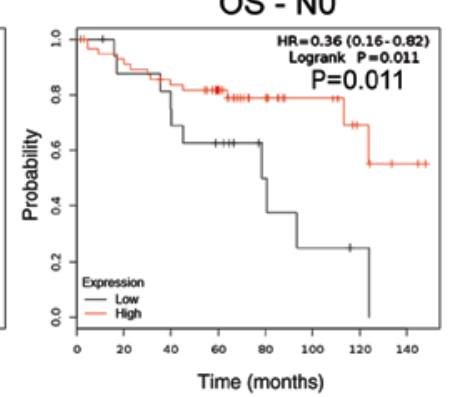

FP - M1

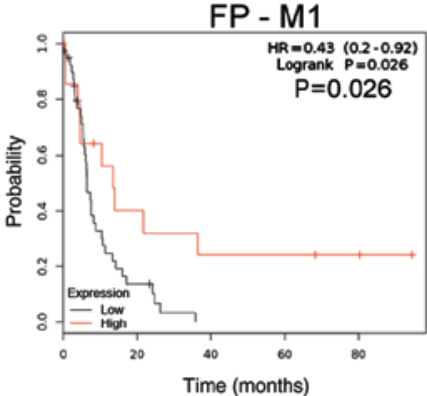

FP - T3

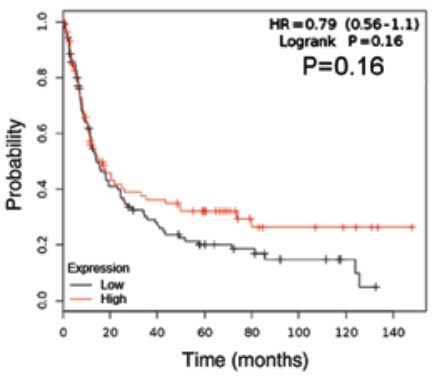

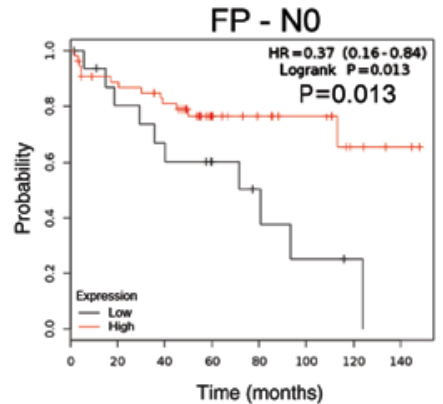

FP - Surgery alone

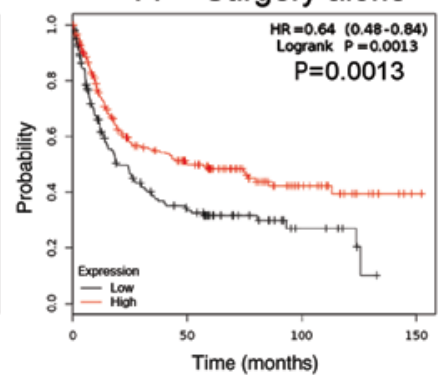

Figure 3. Prognostic values of MLKL in patients with GC. Decreased MLKL was related with poor OS $(\mathrm{HR}=0.80, \mathrm{P}=0.045)$ and FP $(\mathrm{HR}=0.70$, $\mathrm{P}=0.0036)$ in patients with GC. MLKL, mixed lineage kinase domain-like protein; GC, gastric cancer; OS, overall survival; FP, first progression; M0, no distant metastasis; M1, distant metastasis; N0, no lymph node metastasis; N1+2+3, lymph node metastasis; T, depth of invasion.

good OS and poor FP of patients with GC $(\mathrm{OS}, \mathrm{HR}=0.77$, $\mathrm{P}=0.0046$; FP, $\mathrm{HR}=1.34, \mathrm{P}=0.017$; Fig. 5). In patients with lymph node metastasis, higher FA2H expression was associated with longer $\mathrm{OS}$ and $\mathrm{FP}(\mathrm{OS}, \mathrm{HR}=0.59, \mathrm{P}=0.00016$; FP, $\mathrm{HR}=0.67, \mathrm{P}=0.0043$; Fig. 5). By contrast, FA2H had positive effects on OS of patients with GC without lymph node metastasis $(\mathrm{HR}=0.43, \mathrm{P}=0.038$; Fig. 5). Therefore, FA2H and MLKL exhibited similar association with the survival of patients with GC, which suggested that FA2H may be a downstream molecule of MLKL.

\section{Discussion}

MLKL serves important roles in certain malignant tumors, such as pancreatic cancer, ovarian cancer, colon cancer and cervical squamous cell carcinoma (14-17). In patients with cervical squamous cell carcinoma, MLKL expression was increased in cancer tissue compared with normal cervical tissues $(\mathrm{P}=0.004)$ and was negatively correlated with histological grade and lymph node metastasis; low MLKL expression was also associated with poor prognosis (14). Similar conclusions were obtained from 


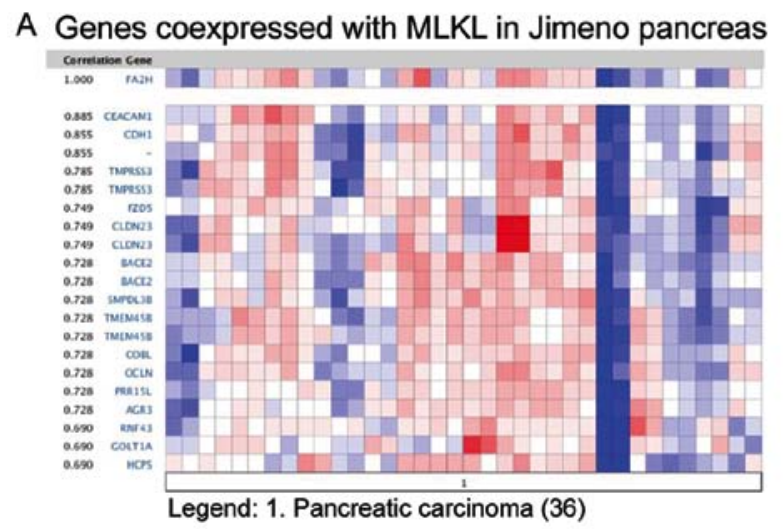

C Genes coexpressed with MLKL in Ooi gastric

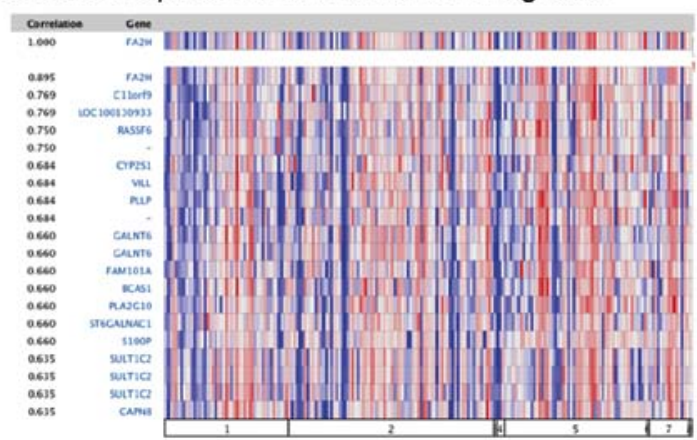

B Genes coexpressed with MLKL in Collisson cellLine

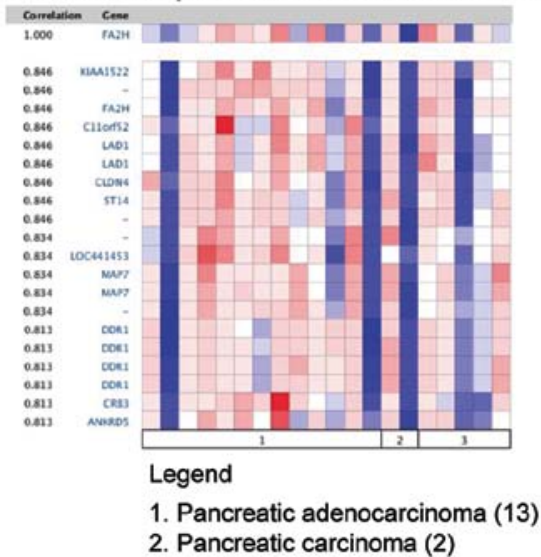

2. Pancreatic carcinoma (2)

\section{Legend:}

1. Diffuse gastric adenocarcinoma (47)

2. Gastric adenocarcinoma (78)

3. Gastric adenosquamous carcinoma (1)

4. Gastric cancer (3)

5. Gastric intestinal type adenocarcinoma (54)

6. Gastric large cell neuroendocrine carcinoma (1)

7. Gastric mixed adenocarcinoma (15)

8. Gastric neuroendocrine neoplasm, NOS (1)

Figure 4. Expression of MLKL was significantly associated with FA2H expression. (A) Genes co-expressed with MLKL in Jimeno Pancreas database. (B) Genes co-expressed with MLKL in Collisson Cell Line database. (C) Genes co-expressed with MLKL in Ooi gastric database. MLKL, mixed lineage kinase domain-like protein; FA2H, fatty acid 2-hydroxylase.
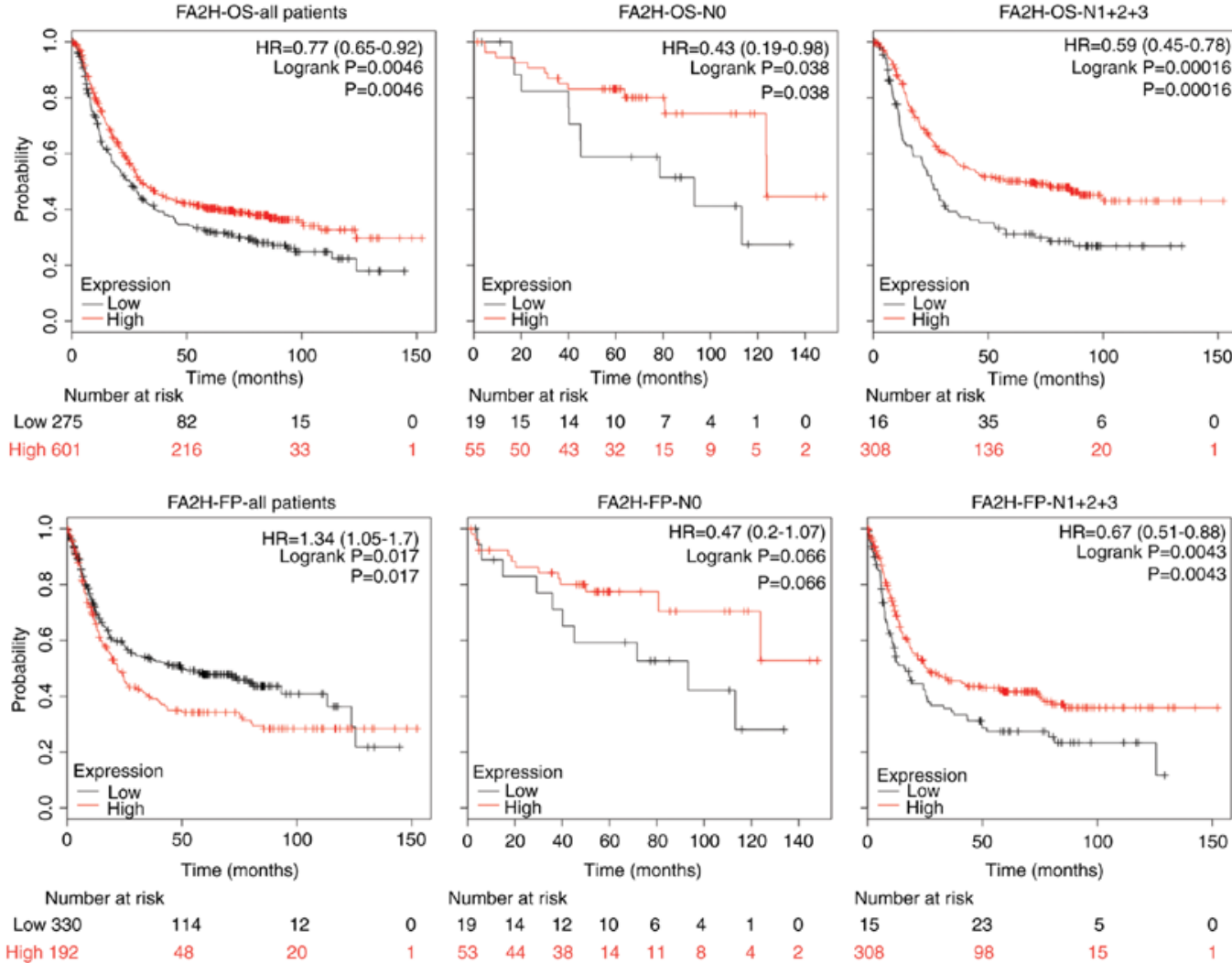

Figure 5. Prognostic values of FA2H in patients with GC. Low FA2H expression levels were associated with poorer OS and FP compared with high FA2H expression levels in patients with GC. FA2H, fatty acid 2-hydroxylase; OS, overall survival; FP, first progression; N0, no lymph node metastasis; N1+2+3, lymph node metastasis. 
studies on early-stage resected pancreatic adenocarcinoma, colon cancer and ovarian cancer (15-17). The results of these studies indicated that MLKL may be a potential prognostic biomarker for patients with carcinoma.

In the present study, MLKL mRNA levels were significantly decreased in GC tissues compared with those in normal tissues. In addition, decreased MLKL was associated with poorer OS and FP in patients with GC. These findings were consistent with those of Ertao et al (21), which demonstrated that loss of MLKL may be involved in GC carcinogenesis and tumor progression, and MLKL may serve as a useful prognosis predictor in patients with GC.

The underlying mechanisms of MLKL in the prognosis of patients with GC are complicated. To the best of our knowledge, phosphorylated MLKL promotes necroptosis, which is a caspase-independent form of regulated cell death $(22,23)$. Necroptosis is mediated by the kinase activities of RIP1 and RIP3 and MLKL (24-28). Deubiquitylation of RIPK1 to RIPK3 induces RIPK3 oligomerization and activation, and the subsequent activation of MLKL, which compromises the integrity of the plasma membrane, leading to the release of intracellular proinflammatory molecules (24). This process underlies the immunogenic nature of necroptotic cancer cells and their ability to induce efficient anti-tumor immunity. When MLKL expression is decreased, cell necroptosis mediated by MLKL is also reduced, leading to continuous proliferation of tumor cells (25-28).

FA2H catalyzes the formation of 2-hydroxy fatty acids, which are precursors for hFA-sphingolipids (29). FA2H is required for the synthesis of hFA-galactolipids in myelin and hFA-ceramide and hFA-glucosylceramide in the epidermis (29). However, the relationship between FA2H and tumors remains unclear. In human cancer cell lines, including Hela, COS-7, HepG2 and A549 (30), FA2H exhibits positive effects on the efficacy of the anti-cancer agent PM02734 (31). Another study has demonstrated that $\Delta 9$-tetrahydrocannabinol induces FA2H expression in human breast cancer MDA MB 231 cells through peroxisome proliferator-activated receptor isoform-selective agonists and antagonists (32). The results of the present study demonstrated that MLKL expression is significantly correlated with FA2H in certain tumors. In addition, FA2H was associated with prognosis of patients with GC. However, no previous studies have reported an association between FA2H and GC or explored the role of FA2H in other tumors.

In conclusion, mRNA expression levels and prognostic values of MLKL in GC were comprehensively analyzed; the results demonstrated that MLKL mRNA expression levels were significantly lower in GC compared with normal tissues, and that patients with GC with low MLKL expression exhibited poorer prognosis compared with patients with high MLKL expression. MLKL may be a prognostic factor for GC; the mechanism of MLKL activity in GC may be through the RIP1/RIP3/MLKL pathway, which participates in cancer cell necrosis and other mechanisms that have not yet been clarified. Further experiments are required to investigate the potential of anti-tumor treatments that target MLKL. In Oncomine co-expression analysis, MLKL expression was significantly correlated with FA2H in some tumors. In addition, FA2 H was associated with the overall survival prognosis in patients with $\mathrm{GC}$; the mechanism of $\mathrm{FA} 2 \mathrm{H}$ affecting the prognosis of patients with GC must be further explored.

\section{Acknowledgements}

Not applicable.

\section{Funding}

No funding was received.

\section{Availability of data and materials}

The datasets generated and/or analyzed during the current study are available in the figshare repository (https://figshare. com/account/articles/8397182).

\section{Authors' contributions}

LS and TH designed the study and applied for Research Ethics Board approval. WS recruited patients and collected data. WY and LS analyzed data and prepared figures. WS wrote the manuscript. All authors approved the final version of the manuscript.

\section{Ethics approval and consent to participate}

This study was approved by the Institutional Review Board of Suzhou University (register ID number: 2016958021). All participants included in this study provided written informed consent.

\section{Patient consent for publication}

Not applicable.

\section{Competing interests}

The authors declare that they have no competing interests.

\section{References}

1. Siegel RL, Miller KD and Jemal A: Cancer statistics, 2019. CA Cancer J Clin 69: 7-34, 2019.

2. Moore MA, Eser S, Igisinov N, Igisinov S, Mohagheghi MA, Mousavi-Jarrahi A, Ozentürk G, Soipova M, Tuncer M and Sobue T: Cancer epidemiology and control in North-Western and Central Asia-past, present and future. Asian Pac J Cancer Prev 11 (Suppl 2): S17-S32, 2010.

3. Chen W, Zheng R, Baade PD, Zhang S, Zeng H, Bray F, Jemal A, Yu XQ and He J: Cancer statistics in China, 2015. CA Cancer J Clin 66: 115-132, 2016.

4. Apicella M, Corso S and Giordano S: Targeted therapies for gastric cancer: Failures and hopes from clinical trials. Oncotarget 8: 57654-57669, 2017.

5. Sun L, Wang H, Wang Z, He S, Chen S, Liao D, Wang L, Yan J, Liu W, Lei X and Wang X: Mixed lineage kinase domain-like proteinmediates necrosis signaling downstream of RIP3 kinase. Cell 148: 213-227, 2012

6. Zhao J, Jitkaew S, Cai Z, Choksi S, Li Q, Luo J and Liu ZG: Mixed lineage kinase domain-like is a key receptor interacting protein 3 downstream component of TNF-induced necrosis. Proc Natl Acad Sci USA 109: 5322-5327, 2012.

7. Vanlangenakker N, Vanden Berghe $\mathrm{T}$ and Vandenabeele $\mathrm{P}$ : Many stimuli pull the necrotic trigger, an overview. Cell Death Differ 19: 75-86, 2012. 
8. Tenev T, Bianchi K, Darding M, Broemer M, Langlais C, Wallberg F, Zachariou A, Lopez J, MacFarlane M, Cain K and Meier P: The ripoptosome, a signaling platform that assembles in response to genotoxic stress and loss of IAPs. Mol Cell 43 432-448, 2011.

9. Coupienne I, Fettweis G and Piette J: RIP3 expression induces a death profile change in U2OS osteosarcoma cells after 5-ALA-PDT. Lasers Surg Med 43: 557-564, 2011.

10. Chu WM: Tumor necrosis factor. Cancer Lett 328: 222-225, 2013

11. Zong WX and Thompson CB: Necrotic death as a cell fate. Genes Dev 20: 1-15, 2006.

12. Zhou Z, Han V and Han J: New components of the necroptotic pathway. Protein Cell 3: 811-817, 2012.

13. Paulsen RD, Soni DV, Wollman R, Hahn AT, Yee MC, Guan A, Hesley JA, Miller SC, Cromwell EF, Solow-Cordero DE, et al: A genome-wide siRNA screen reveals diverse cellular processes and pathways that mediate genome stability. Mol Cell 35 228-239, 2009

14. Ruan J, Mei L, Zhu Q, Shi G and Wang H: Mixed lineage kinase domain-like protein is a prognostic biomarker for cervical squamous cell cancer. Int J Clin Exp Pathol 8: 15035, 2015.

15. He L, Peng K, Liu Y, Xiong J and Zhu FF: Low expression of mixed lineage kinase domain-like protein is associated with poor prognosis in ovarian cancer patients. Onco Targets Ther 6: $1539-1543,2013$.

16. Colbert LE, Fisher SB, Hardy CW, Hall WA, Saka B, Shelton JW, Petrova AV, Warren MD, Pantazides BG, Gandhi K, et al: Pronecrotic mixed lineage kinase domain-like protein expression is a prognostic biomarker in patients with early-stage resected pancreatic adenocarcinoma. Cancer 119: 3148-3155, 2013.

17. Li X, Guo J, Ding AP, Qi WW, Zhang PH, Lv J, Qiu WS and Sun ZQ: Association of mixed lineage kinase domain-like protein expression with prognosis in patients with colon cancer. Technol Cancer Res Treat 16: 428-434, 2017.

18. Ajani JA In H and Sano T: Stomach. In: AJCC Cancer Staging Manual. Amin MB (ed). 8th edition. Springer-Verlag, New York, NY, 2016

19. Xue Y, Ma G, Gu D, Zhu L, Hua Q, Du M, Chu H, Tong N, Chen J, Zhang $\mathrm{Z}$ and Wang M: Genome-wide analysis of long noncoding RNA signature in human colorectal cancer. Gene 556: 227-234, 2015.

20. Livak KJ and Schmittgen TD: Analysis of relative gene expression data using real-time quantitative PCR and the 2(-Delta Delta C(T)) method. Methods 25: 402-408, 2001.
21. Ertao Z, Jianhui C, Kang W, Zhijun Y, Hui W, Chuangqi C, Changjiang Q, Sile C, Yulong $\mathrm{H}$ and Shirong C: Prognostic value of mixed lineage kinase domain-like protein expression in the survival of patients with gastric caner. Tumor Biol 37 13679-13685, 2016.

22. Vandenabeele P, Galluzzi L, Vanden Berghe T and Kroemer G: Molecular mechanisms of necroptosis: An ordered cellular explosion. Nat Rev Mol Cell Biol 11: 700-714, 2010.

23. Krysko O, Aaes TL, Kagan VE, D'Herde K, Bachert C, Leybaert L, Vandenabeele P and Krysko DV: Necroptotic cell death in anti-cancer therapy. Immunol Rev 280: 207-219, 2017.

24. Wang H, Sun L, Su L, Rizo J, Liu L, Wang LF, Wang FS and Wang X: Mixed lineage kinase domain-like protein MLKL causes necrotic membrane disruption upon phosphorylation by RIP3. Mol Cell 54: 133-146, 2014.

25. Dondelinger Y, Declercq W, Montessuit S, Roelandt R, Goncalves A, Bruggeman I, Hulpiau P, Weber K, Sehon CA, Marquis RW, et al: MLKL compromises plasma membrane integrity by binding to phosphatidylinositol phosphates. Cell Rep 7: 971-981, 2014.

26. Zhu Y, Cui H, Gan H, Xia Y, Wang L, Wang Y and Sun Y: Necroptosis mediated by receptor interaction protein kinase 1 and 3 aggravates chronic kidney injury of subtotal nephrectomised rats. Biochem Biophys Res Commun 461: 575-581, 2015.

27. Moriwaki K and Chan FK: RIP3: A molecular switch for necrosis and inflammation. Genes Dev 27: 1640-1649, 2013.

28. Pasparakis M and Vandenabeele P: Necroptosis and its role in inflammation. Nature 517: 311-320, 2015.

29. Alderson NL, Rembiesa BM, Walla MD, Bielawska A, Bielawski J and Hama $\mathrm{H}$ : The human FA2H gene encodes a fatty acid 2-hydroxylase. J Biol Chem 279: 48562-48568, 2004.

30. Alderson NL, Walla MD and Hama H: A novel method for the measurement of in vitro fatty acid 2-hydroxylase activity by gaschromatography-mass spectrometry. J Lipid Res 46: $1569-1575,2005$.

31. Herrero AB, Astudillo AM, Balboa MA, Cuevas C, Balsinde J and Moreno S: Levels of SCS7/FA2H-mediated fatty acid 2-hydroxylation determine the sensitivity of cells to antitumor PM02734. Cancer Res 68: 9779-9787, 2008

32. Takeda S, Ikeda E, Su S, Harada M, Okazaki H, Yoshioka Y, Nishimura H, Ishii H, Kakizoe K, Taniguchi A, et al: $\Delta(9)-\mathrm{THC}$ modulation of fatty acid 2-hydroxylase (FA2H) gene expression: Possible involvement of induced levels of PPAR $\alpha$ in MDA-MB-231 breast cancer cells. Toxicology 326: 18-24, 2014. 